\title{
Mucin expression profile in Barrett's, dysplasia, adenocarcinoma sequence in the esophagus
}

\author{
Burjonrappa SC, Reddimasu S, Nawaz Z, Gao X, Sharma P*, Loggie B \\ Departments of Surgical Oncology and *Pathology, Creighton University. Omaha, NE-68131, USA
}

Correspondence to: Dr. Sathyaprasad C. Burjonrappa, E-mail: sathyabc@yahoo.com

\section{Abstract}

BACKGROUND: The molecular events that accompany the progression to adenocarcinoma (ADC) of the esophagus are poorly understood. Aberrant mucin receptor expression can contribute to increased cell growth and metastatic ability. AIM: The aim of this study was to establish a pattern for mucin (MUC) gene expression in the esophageal mucosa under normal and pathological conditions. SETTING: University Hospital Cancer Center Laboratory. Archived tissue samples studied in a retrospective fashion. MATERIALS AND METHODS: Tissue samples were obtained from the archives of patients with histological evidence of Barrett's esophagus (BE) progressing to ADC. Immunohistochemical analysis was performed using mouse monoclonal antibodies for MUC1, MUC2, MUC5AC, MUC6. Semiquantitative scoring of histological staining was performed using a linear scoring system: 0-staining absent; 1 -staining in 0-25\%; 2-staining in 25-50\%; and 3-staining in 50-75\% of the epithelium. The Binomial test was used to explore trends and differences in frequency of mucin expression along the pathological sequence. RESULTS: Only mild superficial staining of MUC1 was seen in normal squamous epithelium. MUC1 and MUC2 were uniformly expressed in all samples $(7 / 7)$ of $\mathrm{BE}$ and dysplasia $(P=0.008)$. MUC1 expression was upregulated $(7 / 7)$ in progression to adenocarcinoma $(P=0.008)$. The secretory mucins, MUC5AC and MUC6 showed a decrease in expression with progression from BE to dysplasia to ADC $(P<0.05)$. CONCLUSIONS: Downregulation of MUC5AC and MUC6 decreases mucosal protection against gastric acid. Increasing MUC1 expression is associated with progression from dysplasia to ADC. Upregulation of MUC2 reflects intestinal metaplasia in BE.

Key words: Barrett's esophagus, mucin expression

\section{Introduction}

The frequency of adenocarcinoma (ADC) of the esophagus in the western world is increasing at a rate surpassing that of any other cancer. ${ }^{[1]}$ This increase $(8$ $15 \%)$ is largely the result of the growing prevalence of ADC in Barrett's mucosa. It is unclear as to why even after successful fundoplication, Barrett's often persists, committing patients to periodic surveillance endoscopy. Esophageal ADC develops in gastro esophageal reflux in a sequential fashion from Barrett's through various degrees of dysplasia to malignancy, giving a therapeutic window for intervention.

Mucins are high molecular weight glycoproteins synthesized by a broad range of epithelial tissues and are coded for by MUC genes. Mucins are broadly subdivided into two groups: those, which are secreted and form extracellular gels (MUC2, MUC5AC, MUC5B and MUC6) and membrane bound mucins (MUC1, MUC3 and MUC4). Mucins play a key role in the GI tract where the gel not only constitutes a 
physical barrier and lubricant but also generates a protective diffusion barrier for the underlying epithelium. Altered expression of mucin epitopes have been described in Colon and Stomach cancers and correlated with decreased survival. ${ }^{[2-4]}$ Similarly aberrant mucin expression may contribute to continued epithelial damage, increased cell growth and metastatic ability in the esophagus. ${ }^{[5]}$ Jass was the first to suggest that a pattern of mucin staining in Barrett's esophagus may be associated with a greater risk of progression to adenocarcinoma. ${ }^{[6]}$ This study was designed to address the lack of consensus on the role of mucins in the pathological sequence that follows Barrett's disease. We sought to establish a mucin expression pattern that would be valuable diagnostically in the setting of Barrett's disease.

\section{Materials and Methods}

Twenty archived paraffin embedded sections of the esophagus from seven patients with documented histological progression from Barrett's to ADC through the stage of dysplasia were chosen for the study. Barrett's was defined as biopsy proven intestinal metaplasia of any length. The biopsy specimens were collected over a threeyear period from January 2001 to December 2003. Only tissue samples with contiguous evidence of Barrett's, dysplasia and ADC in each cross-section were selected to allow for comparison of MUC gene expression across the pathological sequence. Tissue sections had been stained previously in a standard fashion with hematoxylin and eosin to confirm the diagnosis. ADC was deemed to be arising from the Barrett's esophagus, if, on histological examination, there was intestinal metaplasia in proximity to the tumor.

To limit subjective bias inherent in immunohistochemical measurements at least two samples were obtained from each patient and were studied by two different pathologists. Positive control tissues from gastric fundus, ileum, colon and breast with previously described MUC gene expression patterns, were included with each batch of sections for immunohistochemistry. ${ }^{[7]}$ The primary antibody was omitted as a negative control to test the specificity of the antimucin antibodies for each section. Monoclonal mouse antibodies were used for MUC1, MUC2, MUC5AC and MUC6. Paraffin embedded tissue sections were baked in an oven at 60 degree celsius for thirty minutes and then deparaffinised through three changes of xylene and then rehydrated through a series of decreasing concentrations of ethanol solutions to distilled water. Antigen retrieval was performed by microwave cooking at $121^{\circ} \mathrm{C}$ for 20 minutes in $10 \mathrm{mM}$ citrate buffer, $\mathrm{pH} 6.0$ and then left to cool at room temperature for 60 minutes. Endogenous peroxidase activity was blocked in $1 \%$ hydrogen peroxide in methanol for thirty minutes at room temperature and washed in phosphate buffered solution (PBS) for ten minutes. After blocking nonspecific antibody binding with $10 \%$ normal goat serum in PBS for one hour the slides were incubated overnight with a 1:200 dilution of the primary antibody at $4^{\circ} \mathrm{C}$. Sections were washed three times for ten minutes in PBS and incubated with secondary antibody, goat anti mouse, Vector BA-1000, 1:500 diluted by $5 \%$ normal goat serum in PBS for one hour. Slides were washed three times in PBS. Development was in $0.6 \mathrm{mg} / \mathrm{ml} \mathrm{3-3} \mathrm{diaminobenzidine}$ $(\mathrm{DAB}) / 0.03 \%$ hydrogen peroxide in PBS. Sections were rinsed in water, counterstained with hematoxylin, dipped in saturated lithium carbonate, dehydrated through a series of increasing concentrations of ethanol solutions and mounted under cover-slips. The binomial test was used to explore trends and differences in frequency of mucin and estrogen receptor expression along the pathological sequence. The binomial distribution can be defined as the number of successes produced in a succession of $\mathrm{n}$ independent trials, $P$ being the probability for a success in each trial.

\section{Results}

Expression of the different mucin genes across the pathological sequence in each of the seven patients is outlined in Table 1. A high degree of concordance was

Table 1: Staining pattern for the mucin gene in the transformation from metaplasia to malignancy

\begin{tabular}{lccccc}
\hline & Squamous & Barrett's & Dysplasia & Adenocarcinoma & $P$ \\
\hline MUC I & $7 / 7(100 \%)$ & $7 / 7$ & $7 / 7$ & $7 / 7$ & $0.008^{*}$ \\
\hline MUC II & $0 / 7$ & $7 / 7$ & $7 / 7$ & $5 / 7$ & NS \\
\hline MUC 5AC & $0 / 7$ & $7 / 7$ & $5 / 6(84 \%)$ & $2 / 6(33 \%)$ & $<0.05$ \\
\hline MUC 6 & $0 / 7$ & $7 / 7$ & $5 / 7(72 \%)$ & $3 / 7(42 \%)$ & $<0.05$ \\
\hline
\end{tabular}

*Intensity of MUC1 expression: MUC I expression was noted in 0-25\% of Barrett's, $25-50 \%$ of dysplastic epithelium and $50-75 \%$ of adenocarcinoma. TABLE I Shows declining secretory mucin (MUC 5AC, MUC 6) expression as one progresses towards malignancy and persistent expression of membrane bound mucin (MUC I) although of increasing intensity. 
noted in the interpretation of the stains by each of the pathologists. Results of MUC gene expression in control tissues were similar to previously published data. ${ }^{[8]}$ There was no evidence of nonspecific staining by primary antibodies in the negative controls and no positive signals were seen in non epithelial cells for any of the mucin stains.

Results for MUC gene expression were divided into secretory and membrane bound mucins. In the normal squamous epithelium MUCl, a membrane bound mucin, was expressed along the surface in a superficial manner. With progression along the pathological sequence the intensity of expression in the linear scale increased. There was staining in $0-25 \%$ in normal epithelium to $25-50 \%$ in dysplasia and $50-75 \%$ staining in adenocarcinoma $(P=0.008)$ [Figures 1-3]. Secretory mucins were not found in the normal squamous epithelium. In metaplastic cells where it was expressed

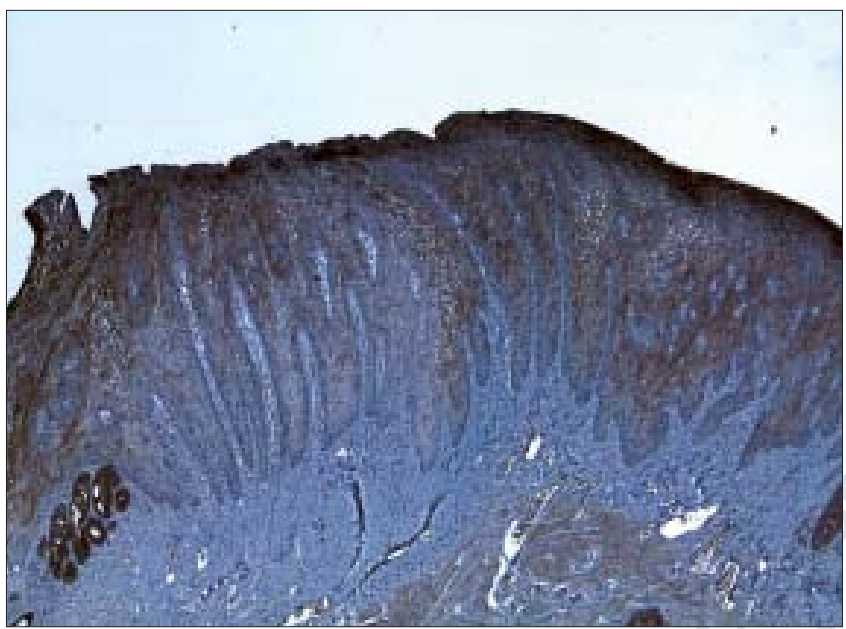

Figure 1: Superficial staining with MUC1 in Barrett's. Immunohistochemistry stains magnification: x20

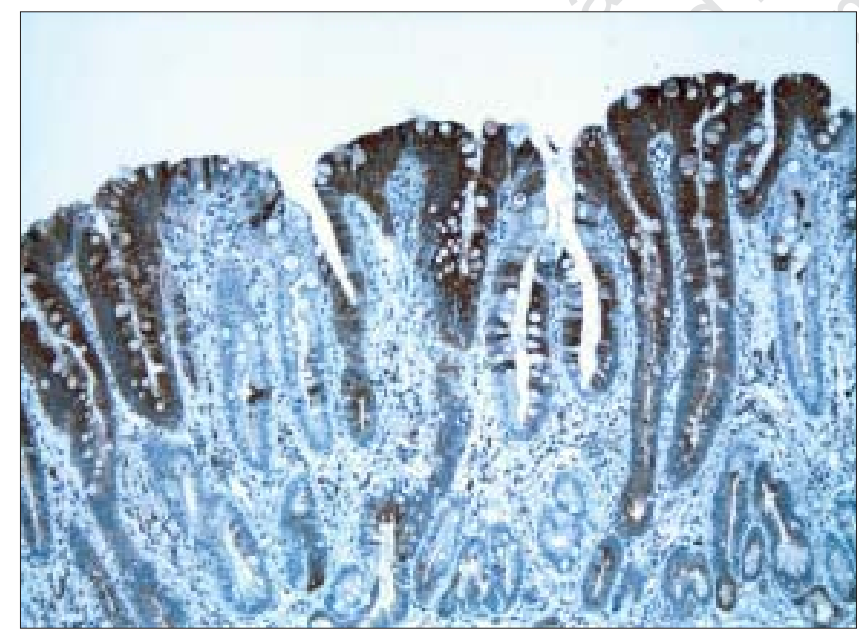

Figure 2: Staining with MUC1 in Barrett's with dysplasia. Immunohistochemistry stains magnification: x20

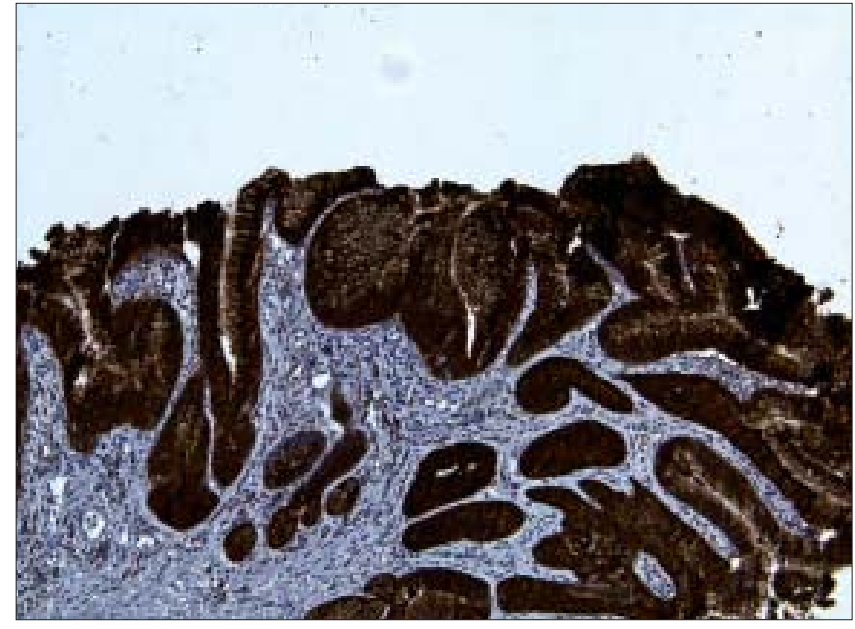

Figure 3: Increased intensity of MUC1 staining in adenocarcinoma. Immunohistochemistry stains magnification: x20

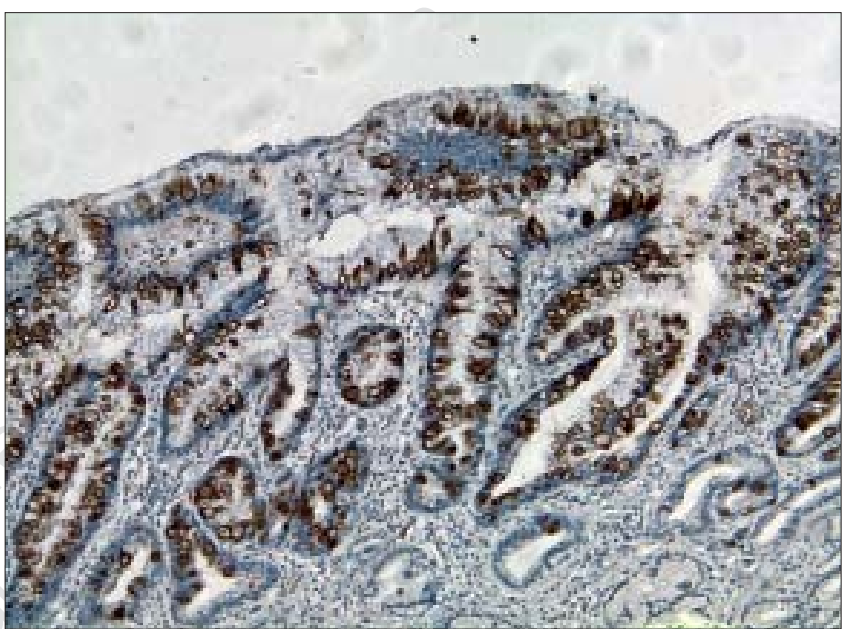

Figure 4: MUC2 staining in goblet cells. Immunohistochemistry stains magnification: $x 20$

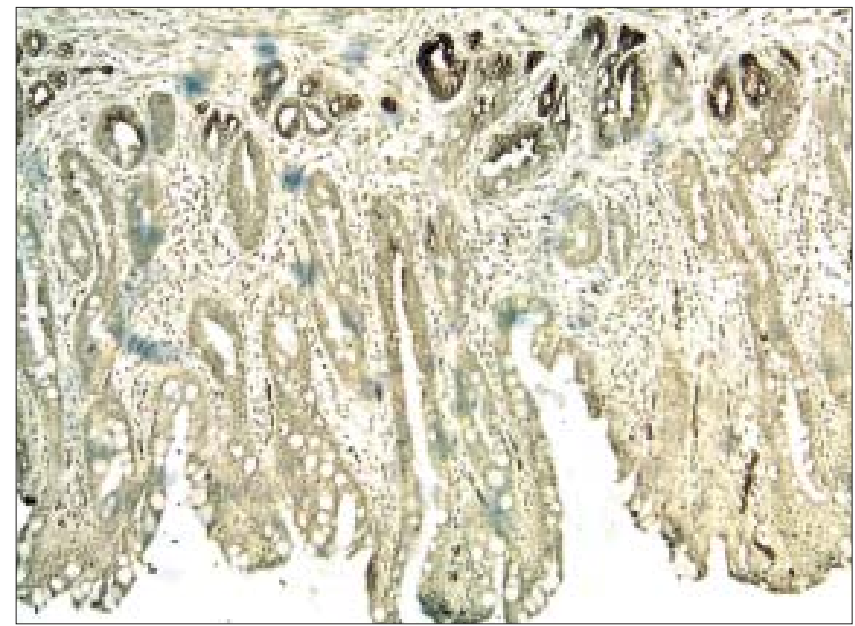

Figure 5: MUC6 staining in deep foveolar glands. Immunohistochemistry stains magnification: $\mathbf{x 2 0}$ 
the MUC2 gene was confined to the goblet cells [Figure 4]. In Barrett's and dysplasia the expression was $7 / 7(100 \%)$ while in adenocarcinoma the expression proportionately decreased to $5 / 7(71 \%)$. MUC5AC was found in the foveola of superficial epithelium and MUC6 in the deep glands of the metaplastic epithelium [Figure 5]. While there was an expression of $7 / 7$ $(100 \%)$ of both these gastric secretory mucins in Barrett's, their expression proportionately decreased to $5 / 6(84 \%)$ and $5 / 7(71 \%)$ in dysplasia and 2/6 (33\%) and $3 / 7(42 \%)$ respectively in adenocarcinoma $(P<0.05)$.

\section{Discussion}

A high degree of correlation between mucin mRNA detection by in situ hybridization and mucin immunohistochemistry has been established. ${ }^{[9]}$ The latter is quicker and needs a less sophisticated laboratory. Specific and efficient antibody tests have been validated for all apomucins studied in this article allowing us to perform experiments over a reasonable time frame and draw accurate conclusions as compared to previously available tests.

MUCl expression showed an up-regulation along the metaplasia, dysplasia and adenocarcinoma sequence in this study. In a recent study MUCl positive Barrett's cases showed a trend toward longer length of Barrett's compared with MUCl negative cases. ${ }^{7]}$ The MUCl peptide core mediates firm adhesion of tumor cells to adjacent cells via binding to intercellular adhesion molecule-1 (ICAM-1) and facilitates metastases. [10] Binding to ICAM-1 also impairs T-lymphocyte surveillance. ${ }^{[1]}$ Abnormal oncogene expression has been reported in Barrett's epithelium, including c-erbB2, p53 and primary cell nuclear antigen. An association between c-erbB2 and MUCl has been hypothesized. ${ }^{[12]}$ Thus, over expression and abnormal glycosylation of MUCl sets the stage for increasing cellular instability.

It is generally acknowledged that the "metaplastic epithelium" may reflect an adaptive response to a new luminal environment with MUC5AC and MUC6 offering protection from gastric acid and MUC2 associated with protection from bile. In this study, we noted a significant down-regulation of these secretory mucins as one progressed along the metaplasiaadenocarcinoma sequence. The trefoil peptides (TFF1, TFF2) are associated with mucosal repair and act synergistically with mucins (MUC5AC) to protect epithelial tissues. ${ }^{[13]}$ Downregulation of secretory mucins thus could impair mucosal protection. Loss of MUC2 in previously mucin secreting cells may indicate loss of differentiation in the neoplastic cells as illustrated by a decrease in expression in adenocarcinoma.

In all intestinal metaplasia samples, we found gastrictype MUC gene expression, suggesting a common origin for gastric and intestinal metaplasia. Others have also noted the possibility of activation of multipotent esophageal stem cells following destruction of normal squamous epithelium. ${ }^{[14,15]}$ At present it is however unclear as to whether gastric metaplasia and intestinal metaplasia develop simultaneously or successively. There appears to be a definite order in the appearance and subsequent decrease of the various mucins in the Barrett's-adenocarcinoma sequence. It is still not clear whether this is the primary cause for the persistence of the Barrett's epithelium in-spite of successful anti-reflux surgery, an adaptive response to reflux or if it reflects the genetic instability associated with this preneoplastic condition. Further studies in patients undergoing surveillance for Barrett's and dysplasia will help answer whether mucin gene expression has a diagnostic role in predicting those at risk and does not merely represent an artifact of progression. An open question is whether therapeutic manipulation of MUC gene expression will decrease the risk of malignancy for patients with $\mathrm{BE}$ and dysplasia.

\section{References}

1. Devesa SS, Blot WJ, Fraumeni JF Jr. Changing patterns in the incidence of esophageal and gastric carcinoma in the United States. Cancer 1998;83:2049-53.

2. Ho SB, Chandler DJ, Logan G, Toribara NW. Intestinal and gastric mucin core peptide expression in colon cancer: Correlation with histology, stage and survival. Gastroenterology 1993;104:A410.

3. Ho SB, Shekals LL, Neihans GA, Lyftgot CT. Human gastric mucin: Gene heterogeneity and alterations in gastric neoplasia. Gastroenterology 1993;104:A410.

4. Ho SB, Niehans GA, Lyftogt C, Yan PS, Cherwitz DL, Gum ET, et al. Heterogenity of mucin gene expression in normal and neoplastic tissues. Cancer Res 1993;53:641-51.

5. Ogata S, Maimonis PJ, Itzkowitz SH. Mucins bearing the cancer associated sialosyl Tn antigen mediate inhibition of natural killer cell toxicity. Cancer Res 1992;52:4741-6.

6. Jass JR. Mucin histochemistry of columnar epithelium of the esophagus: A retrospective study. J Clin Pathol 1981;34:866-70.

7. Glickman JN, Shahsafaei A, Odze RD. Mucin core peptide expression can help differentiate Barret's esophagus from intestinal metaplasia of the stomach. Am J Surg Pathol 2003;27:1357-65.

8. Audie JP, Janin A, Porchet N, Copin MC, Gosselin B, Aubert JP. Expression of human mucin genes in respiratory, digestive and reproductive tracts ascertained by in-situ hybridization. J Histochem Cytochem 1993;41:1479-85.

9. Arul GS, Moorghen M, Myerscough N, Alderson DA, Spicer RD, Corfield AP. Mucin gene expression in Barrett's esophagus: An in situ hybridization and Immunohistochemical study. Gut 2000;47:753-61.

10. Rahn JJ, Shen Q, Mah BK, Hugh JC. MUC1 initiates a calcium signal after ligation by intercellular adhesion molecule-1. J Biol Chem 2004;279:29386-90. 
11. van de Wiel-van Kemenade E, Ligtenberg MJ, de Boer AJ, Buijs F, Vos HL, Melief CJ, et al. Episialin (MUC1) inhibits cytotoxic lymphocyte-target cell interaction. J Immunol 1993;151:767-76.

12. Jaskiewicz J, Coghill G, Hopwood D, Wormsley KG. Oncogenes and onco-suppressor gene in adenocarcinoma of the esophagus. Gut 1992;33:1033-8.

13. Labouvie C, Macahdo JC, Carneiro F, Sarbia M, Vieth M, Porschen $\mathrm{R}$, et al. Differential expression of mucins and trefoil peptides in native epithelium, Barrett's metaplasia and squamous cell carcinoma of the esophagus. J Cancer Res Clin Oncol 1999; 125:71-6.

14. Shields HM, Sawhney RA, Zwas F, Boch JA, Kim S, Goran D, et al.
Scanning electron microscopy of the human esophagus: Application to Barrett's esophagus: A precancerous lesion. Microsc Res Tech 1995;31:248-56.

15. Guillem P, Billeret V, Buisine MP, Flejou JF, Lecomte-Houcke M, Degand $\mathrm{P}$, et al. Mucin gene expression and cell differentiation in human normal, premalignant and malignant esophagus. Int J Cancer 2000;88:856-61.

Source of Support: Nil, Conflict of Interest: None declared.

\section{Announcement}

\section{Free access to the Cochrane Library for everyone in India}

Anyone in India with access to the Internet now has complementary access to reliable, up-to-date evidence on health care interventions from The Cochrane Library, thanks to sponsorship provided by the Indian Council of Medical Research (ICMR) that recently signed a three-year contract for a national subscription with the publishers, John Wiley \& Sons.

The Cochrane Library (available at www.thecochranelibrary.com) is considered by many to be the single most reliable source for evidence on the effects of health care interventions. It includes seven databases that are updated quarterly, four of which are the efforts of the 15,000 international contributors of the Cochrane Collaboration (www.cochrane.org). The Cochrane Database of Systematic Reviews currently contains 4655 regularly-updated systematic reviews and protocols of reviews in preparation.

The Cochrane Controlled Trials Register currently contains references, mostly with abstracts, of more than 48,900 controlled clinical trials-easily the largest collection of such trials in the world.

The Cochrane Database of Methodology Reviews contains 22 systematic reviews of the science of reviewing evidence. The Cochrane Methodology Register contains the bibliography of 9048 articles that could be relevant to anyone preparing systematic reviews.

The three other databases in The Cochrane Library include the:

- Database of Abstracts of Reviews of Effectiveness, summaries of 5931 systematic reviews published elsewhere and quality appraised by the UK National Health Service (NHS) Centre for Reviews and Dissemination.

- Health Technology Assessment Database that contains details of 6358 completed and ongoing health technology assessments.

- NHS Economic Evaluation Database that contains 20,292 abstracts of quality assessed economic evaluations from around the world.

Also available is information about the Cochrane Collaboration. One can search for interventions or health conditions across all these databases using free text terms or medical subject headings (MeSH).

From 29 January 2007 the Cochrane Library is freely available to all residents of India with Internet access thanks to funding from the Indian Council of Medical Research (ICMR) (www.ICMR.nic.in), and work of the South Asia Cochrane Network (www.cochrane-sacn.org). 